\title{
AVALIAÇÃO DE CARACTERÍSTICAS DE BIODIESEIS DE FONTES ALTERNATIVAS SUBMETIDOS A CONDIÇÕES DE ARMAZENAGEM DIFERENCIADA
}

\author{
Maria Juliane Suota ${ }^{a}$, Edesio Luiz Simionatto ${ }^{a}$, , Dilamara Riva Scharf ${ }^{a}$, Valnice Motta ${ }^{\text {a }}$, Daniela Moser ${ }^{\mathrm{a}}$, Luciano Basto \\ Oliveira $^{\mathrm{b}}$, Luiz Roberto Martins Pedroso ${ }^{\mathrm{b}}$, Alberto Wisniewski Jrc $^{\mathrm{c}}$, Vinicyus Rodolfo Wiggers ${ }^{\mathrm{d}}$, Vanderleia Botton ${ }^{\mathrm{d}}$ e Henry \\ França Meier ${ }^{d}$
}

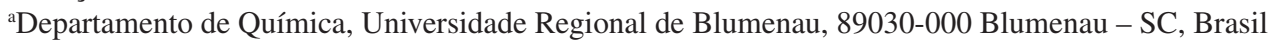

${ }^{\mathrm{b} E C O 100 ~-~ S u s t a i n a b l e ~ D e v e l o p m e n t ~ L t d a ~}$

'Departamento de Química, Universidade Federal de Sergipe, 49100-000 São Cristóvão - SE, Brasil

${ }^{\mathrm{d}}$ Departamento de Engenharia Química, Universidade Regional de Blumenau, 89030-000 Blumenau - SC, Brasil

Recebido em 16/02/2018; aceito em 12/03/2018; publicado na web em 29/03/2018

\begin{abstract}
EVALUATION OF BIODIESEL CHARACTERISTICS OF ALTERNATIVE SOURCES SUBMITTED TO DIFFERENTIAL STORAGE CONDITIONS. Biodiesel is an alternative fuel obtained from different biomass sources and processes. Nowadays, this biofuel has gained importance due to environmental concerns and rising energy demand. In the biodiesel production chain, stability is a critical issue, as it is linked to the maintenance of its original properties. This work performed a study evaluating the influence of storage conditions on the quality aspects of biodiesel. Thus, different samples of biodiesel obtained from soybean and frying oils were produced and placed in containers made by plastic, glass and tin, and stored in presence or absence of an oxidative atmosphere, moisture and light. These samples were analyzed to evaluate the ester content, acidity, iodine value, kinematic viscosity and oxidative stability. The characterization with GC-MS and GC/FID were done aiming to determine the chemical composition. Blends with frying biodiesel into soybean biodiesel have also been produced in concentrations ranging from $1 \%, 5 \%$ and $10 \%(\mathrm{~m} / \mathrm{m})$ in order to evaluate the mixing effect. In the initial storage time, all tested parameters were in accordance to the legislation. Distinct behaviors were identified for each sample according to the container type, being the glass the most effective of them in maintenance of biodiesel characteristics.
\end{abstract}

Keywords: biodiesel; storage conditions; quality; oxidative stability.

\section{INTRODUÇÃO}

Biodiesel é uma alternativa energética de origem renovável que vem apresentando características ímpares quando comparado aos combustíveis convencionais. Sua versatilidade permite que ele seja obtido por meio das mais variadas fontes de oleaginosas em diferentes regiões do mundo. Em geral, o biodiesel produzido a partir de óleos vegetais consiste em uma mistura de ésteres de ácidos graxos com um número de átomos de carbono de $\mathrm{C}_{14: 0}$ até $\mathrm{C}_{22: 0}$, com vários níveis de insaturação. ${ }^{1-3}$ A corrida pela substituição dos derivados fósseis devido às especulações sobre a escassez, ameaças constantes de elevação de preços e principalmente por preocupações com as emissões de gases nocivos ao ambiente têm estimulado o processamento de diversos materiais graxos, que por sua vez têm produzido biodieseis de qualidade apreciável. ${ }^{4-7}$ Ao biodiesel, são atribuídas algumas vantagens, tais como ser obtido de fontes renováveis, capazes de promover o desenvolvimento regional e sustentável, a biodegradabilidade, a diminuição na emissão de gases do efeito estufa, a não toxicidade, a ausência de compostos aromáticos, o elevado número de cetano, além da alta lubricidade em comparação ao petróleo. ${ }^{5,8-10}$

Os principais empecilhos em relação à produção de biodiesel são atribuídos ao custo da matéria-prima, à competição com a produção de alimentos e à sua estabilidade à oxidação. ${ }^{4,5}$ No entanto, outras opções de óleos e gorduras, que não competem com a cadeia alimentícia nem tampouco são onerosos como os óleos virgens tradicionalmente utilizados são matérias-primas atrativas para a produção de biodiesel. Entre as misturas graxas mais promissoras destacam-se o óleo de pinhão-manso, o lodo gorduroso de esgoto, as gorduras e sebos

*e-mail: edesiofurb@gmail.com oriundos de abatedouros, os óleos de fritura residuais e uma variedade de óleos de espécies não comestíveis..$^{4-7,9,11-14}$ Essas alternativas oferecem a possibilidade de aumentar a oferta de biodiesel a partir de matérias-primas economicamente mais acessíveis, sem interferir na produção de alimentos. Conforme supracitado, o outro fator crítico da produção de biodiesel é a estabilidade à oxidação. Esta se refere à tendência dos combustíveis em reagir com o oxigênio em temperaturas próximas à do ambiente. ${ }^{15}$ Já a estabilidade à estocagem é definida conforme a capacidade de resistência do biocombustível às mudanças físicas e químicas ocasionadas em decorrência da sua interação com o ambiente. ${ }^{16} \mathrm{O}$ biodiesel é muito sensível à exposição ao ar e à luz, a temperaturas elevadas, à presença de traços de metais com ação catalítica à oxidação, pigmentos, quantidade de insaturações e até mesmo o recipiente no qual o combustível é armazenado pode estar relacionado com a preservação das suas propriedades originais. ${ }^{11,17-20}$ Atualmente, a conservação do biodiesel é realizada por meio da adição de antioxidantes sintéticos ou naturais, os quais mantêm as propriedades desse biocombustível dentro das especificações. Uma ampla variedade de antioxidantes já foi testada e tem produzido resultados promissores na garantia da integridade das moléculas de éster. ${ }^{21-25}$

Nesse contexto, vários pesquisadores vêm conduzindo estudos relacionados à estabilidade de biodiesel para identificar os fatores que provocam sua oxidação, aperfeiçoar os métodos de avaliação da estabilidade oxidativa, bem como aprimorar a rota de síntese e as condições de estocagem, na tentativa de manter a qualidade do combustível. ${ }^{11,16,18-20,25-28}$ A degradação do biodiesel pode ser identificada por meio de alterações nas suas propriedades, tais como o índice de acidez, índice de peróxido, teor de ésteres e índice de iodo, além da viscosidade, densidade, número de cetano e poder calorífico. ${ }^{29}$ Outros indicadores de deterioração dos biocombustíveis estão associados à 
sua coloração e à presença de produtos de oxidação que podem ser corrosivos ao motor e inclusive levar ao entupimento das bombas e filtros de injeção devido ao aumento da viscosidade..$^{17,18,30}$

Considerando a susceptibilidade do biodiesel às reações químicas em consequência da sua natureza insaturada e levando em conta ainda os fatores externos aos quais é vulnerável, este trabalho procurou estudar a influência das diferentes condições de armazenagem sob algumas de suas propriedades. Assim sendo, o primeiro passo da pesquisa foi sintetizar o biodiesel a partir de duas matérias-primas distintas (o óleo de soja virgem e o óleo de fritura) e, por conseguinte, avaliar o comportamento de suas principais propriedades na forma pura e de misturas ao longo do período de estocagem. Também foi realizado monitoramento das propriedades de um biodiesel produzido em escala piloto (a partir de óleo de caixa de gordura) em comparação com as amostras obtidas nesta pesquisa.

\section{PARTE EXPERIMENTAL}

\section{Amostras de biodiesel}

O biodiesel obtido a partir do óleo da caixa de gordura foi fornecido pela empresa ECO100 Desenvolvimento Sustentado LTDA, produzido em escala piloto, na planta instalada na Estação de Tratamento de Alegria (ETE Alegria), localizada no Bairro do Caju - RJ. Os biodieseis de óleo de fritura e de óleo virgem foram obtidos por transesterificação em reator apropriado, nas dependências do Laboratório de Desenvolvimento de Processos do Departamento de Engenharia Química da Universidade Regional de Blumenau FURB, cidade de Blumenau - SC.

$\mathrm{Na}$ transesterificação por catálise básica, juntaram-se ao reator 3,68 kg do óleo e 27,64 g de KOH P.A. (mín. 85\%), diluídos em 4 L de metanol P.A. (mín. 99,5\%), a razão molar álcool:óleo:catalisador foi de 23,66:1,00:0,12. A mistura reacional ficou sob agitação por duas horas a $60{ }^{\circ} \mathrm{C} .{ }^{31} \mathrm{Em}$ seguida, o metanol excedente foi retirado por meio de destilação simples e o restante da reação (biodiesel + glicerina) ficou em repouso por 24 horas para decantação da glicerina. Após a retirada da glicerina, fez-se a primeira lavagem do biodiesel, utilizando $20 \%$ de água em relação à massa de biodiesel e 0,25\% de ácido clorídrico P.A. (37,7\%) em relação à massa de água. Manteve-se tudo no interior do reator em agitação por 15 minutos a $60{ }^{\circ} \mathrm{C}$. Após este tempo, aguardou-se uma hora para a decantação. A água de lavagem apresentou $\mathrm{pH}$ 2, e um novo processo de lavagem utilizando apenas água destilada foi realizado. Verificou-se que a água da segunda lavagem apresentou pH 8. A massa do biodiesel obtido foi determinada, e o produto foi submetido então à secagem em estufa a $120^{\circ} \mathrm{C}$ por 4 horas.

\section{Misturas}

Misturas de biodiesel de óleo de fritura foram realizadas com $1 \%$, $5 \%$ e $10 \%(\mathrm{~m} / \mathrm{m})$ em biodiesel de óleo de soja. Essas misturas foram armazenadas em frascos de vidro âmbar, sob atmosfera de nitrogênio e avaliadas em seus parâmetros físico-químicos num período de sessenta dias. Os parâmetros avaliados foram o índice de acidez (ABNT NBR 14448:2009), a estabilidade à oxidação (BS EN 14112:2003) e o teor de ésteres (baseada na BS EN 14103:2003).

\section{Determinação da composição química dos óleos}

A composição química dos óleos utilizados neste trabalho foi realizada por intermédio do processo de derivatização e conversão dos triacilglicerídeos e ácidos graxos livres em seus respectivos ésteres. Para a derivatização dos óleos, uma gota da amostra, $1 \mathrm{~mL}$ de metanol e uma gota de $\mathrm{H}_{2} \mathrm{SO}_{4}$ concentrado foram adicionados em um frasco com tampa. A mistura foi agitada e, em seguida, levada à estufa a $60{ }^{\circ} \mathrm{C}$ por 30 minutos. Após atingir a temperatura ambiente, foi acrescentado $1 \mathrm{~mL}$ de $n$-heptano e $2 \mathrm{~mL}$ de $\mathrm{NaCl}_{(\mathrm{aq})}$ a $10 \%$. A fase orgânica foi recolhida e submetida à análise por cromatografia em fase gasosa acoplada à especrometria de massas (CG-EM). ${ }^{32}$ Para a caracterização, foi empregada a metodologia baseada no trabalho de Wisniewski e colaboradores, utilizando o cromatógrafo a gás CGEM, modelo QP2010PLUS Shimadzu, equipado com coluna Rtx5 (30 m x 0,25 mm x 0,1 $\mu \mathrm{m}$ ) e hélio 5.0 analítico como gás de arraste em fluxo constante de $1 \mathrm{~mL} \mathrm{~min}{ }^{-1}$. A temperatura do injetor foi de $250{ }^{\circ} \mathrm{C}$, operando no modo split (1:50) com volume de injeção de $1 \mu \mathrm{L}$. A programação de temperatura do forno iniciou em $60^{\circ} \mathrm{C} \mathrm{min}{ }^{-1}$ por $5 \mathrm{~min}$, com aumento de $5{ }^{\circ} \mathrm{C} \mathrm{min}{ }^{-1}$ até $280{ }^{\circ} \mathrm{C}$, permanecendo por 10 minutos. A temperatura da fonte de íons foi de $250{ }^{\circ} \mathrm{C}$ e da interface de $300^{\circ} \mathrm{C} .{ }^{33}$

Para a análise da presença de ácidos graxos livres nos biodieseis, foi preparada uma solução de $500 \mu \mathrm{g} \mathrm{mL}^{-1}$ de biodiesel em tetraidrofurano, acrescida de $100 \mu \mathrm{L}$ do derivatizante $N$-Metil- $N$ - (trimetilsilil) trifluoroacetamida (MSTFA). Nesta etapa, foram empregadas as mesmas condições descritas na metodologia. ${ }^{33}$

\section{Condições de armazenagem do biodiesel}

Os biodieseis de soja e de fritura foram submetidos a três diferentes tipos de frascos de armazenamento. Em frascos fechados e mantidos a temperatura ambiente, de plástico (polietileno), de vidro âmbar e de lata (folha de flandres). O biodiesel de caixa de gordura foi armazenado nos mesmos recipientes, mas com o diferencial de ser estocado com e sem purga de nitrogênio. Os frascos de plástico e vidro utilizados possuíam capacidade de $100 \mathrm{~mL}$, já as latas capacidade de $500 \mathrm{~mL}$. Foram colocados $100 \mathrm{~mL}$ de biodiesel em cada frasco.

\section{Teor de ésteres}

O teor de ésteres foi determinado por CG/DIC baseando-se na norma europeia BS EN 14103:2003. O padrão interno utilizado foi o heptadecanoato de metila em heptano, na concentração de $10 \mathrm{mg} \mathrm{mL}^{-1}$. Cada amostra de biodiesel $(50 \mathrm{mg})$ foi dissolvida em $1 \mathrm{~mL}$ da solução de padrão interno. O cromatógrafo empregado foi o Shimadzu CG-2010 com Auto Injetor AOC-5000 e coluna STABILWAX (30 m x 0,25 mm x 0,25 $\mu \mathrm{m}$ ). Utilizou-se como gás de arraste o hélio 5.0 analítico com velocidade linear de $45 \mathrm{~cm} \mathrm{~s}^{-1}$. As temperaturas do injetor e do detector foram $250{ }^{\circ} \mathrm{C}$ e $280{ }^{\circ} \mathrm{C}$, respectivamente. A razão do split foi de 1:50 e a temperatura do forno (isoterma) se manteve em $210^{\circ} \mathrm{C}$ por 40 minutos. $\mathrm{O}$ volume de amostra injetado foi de $1 \mu \mathrm{L}$.

\section{Características físico-químicas}

A estabilidade à oxidação e o índice de iodo foram realizados seguindo as normas europeias BS EN 14112:2003 e BS EN 14111:2003, respectivamente. O índice de acidez foi avaliado com base na norma ABNT NBR 14448:2009. A determinação da viscosidade cinemática foi feita segundo a norma ASTM D 445:2006.

\section{RESULTADOS E DISCUSSÃO}

A estabilidade de um biodiesel é a resistência deste a um conjunto de modificações químicas que são inerentes ao processo de estocagem por longos períodos. O contato com o ar, calor e água são os principais fatores que afetam a estabilidade ao armazenamento. ${ }^{34}$ Este trabalho avaliou alguns dos principais parâmetros indicadores de estabilidade 
do biodiesel que podem ser alteradas no processo de estocagem. Os recipientes utilizados foram frascos de plástico e vidro com capacidade de $100 \mathrm{~mL}$, e lata com capacidade de $500 \mathrm{~mL}$. Sendo que o volume de biodiesel armazenado nos três recipientes foi de $100 \mathrm{~mL}$.

\section{Caracterização dos óleos}

A composição percentual relativa do óleo de soja virgem e de óleo de fritura está apresentada na Tabela 1.

A composição dos óleos de soja e de fritura apresentaram os mesmos ácidos graxos, variando em seu percentual relativo. $\mathrm{O}$ biodiesel de óleo de fritura apresentou $80,63 \%$ de compostos insaturados e o de soja $84,71 \%$, indicando degradação das insaturações.

\section{Rendimento do processo de transesterificação}

Após cada reação, foram recuperados 2,36 kg de biodiesel de óleo virgem e 2,38 kg de biodiesel de óleo de fritura. Obtiveram-se $0,62 \mathrm{~kg}$ de glicerina para o biodiesel de óleo de soja virgem e 0,66 kg para biodiesel de óleo de fritura.

\section{Teor de ésteres}

O teor de ésteres dos biodieseis de óleo de soja virgem e de fritura foi determinado no início do período de armazenamento e, após o acondicionamento nos respectivos recipientes, este parâmetro foi avaliado a cada 30 dias. A Resolução ANP N ${ }^{\circ} 14$ de 11.05.2012 estabelece que o teor mínimo de ésteres é de $96,5 \%$.
Os dados da Tabela 2 mostram que os ésteres preparados a partir do óleo de soja virgem e do óleo de fritura variaram o seu teor de éster ao longo da estocagem, sendo notório que a armazenagem em lata apresentou a decomposição mais intensa em ambos os biodieseis. Uma hipótese para este fato é o não preenchimento total da lata, que permitiu a existência de uma camada gasosa no espaço excedente e, por exemplo ar atmosférico, e por isso, houve maior interação do biocombustível com o recipiente ou reação de oxidação catalizada pela superfície metálica.

Os valores de teor de ésteres do biodiesel de caixa de gordura estão listados na Tabela 3. A amostra de biodiesel de óleo de caixa de gordura foi avaliada também quanto à presença ou ausência de uma atmosfera oxidativa.

A amostra de biodiesel de caixa de gordura obteve maior alteração do teor de ésteres quando exposta à atmosfera oxidativa. Com relação à estocagem, o vidro, principalmente na ausência de nitrogênio, foi o recipiente que melhor conservou as propriedades e a composição dos biodieseis. A embalagem metálica, mesmo em atmosfera de gás inerte, apresentou os menores teores de ésteres. Segundo estudos envolvendo a reação de oxidação de amostras de biodiesel de fontes alternativas, o efeito catalisador de metais, como cobre, confirma a influência na modificação e degradação da estrutura de ésteres derivados de ácidos graxos, especialmente nas insaturações. ${ }^{15}$ Após cento e oitenta dias de armazenamento, observou-se que os teores de ésteres do biodiesel de óleo de soja virgem armazenado em recipiente lata passou de $81,95 \%$ para $48,8 \%$ em massa, o que pode ser explicado pelo efeito catalisador do metal da lata, além do volume de $400 \mathrm{~mL}$ não preenchido com biodiesel.

Tabela 1. Composição química do óleo de soja virgem e de fritura

\begin{tabular}{|c|c|c|c|c|c|}
\hline \multirow{2}{*}{ Pico } & \multirow{2}{*}{ Éster metílico } & \multicolumn{2}{|c|}{ Óleo de fritura } & \multicolumn{2}{|c|}{ Óleo de soja virgem } \\
\hline & & Tempo de retenção (min.) & $\% *$ & Tempo de retenção (min.) & $\% *$ \\
\hline 1 & Hexadecanoico $\mathrm{C}_{16: 0}$ & 32,667 & 11,790 & 32,670 & 12,140 \\
\hline 2 & Linoleico $\mathrm{C}_{18: 2}$ & 35,841 & 50,300 & 35,856 & 53,220 \\
\hline 3 & Oleico $\mathrm{C}_{18: 1}$ & 35,958 & 30,330 & 35,969 & 31,490 \\
\hline 4 & Octadecanoico $\mathrm{C}_{18: 0}$ & 36,441 & 3,127 & 36,443 & 1,720 \\
\hline Total & & & 95,547 & & 98,570 \\
\hline
\end{tabular}

*Percentual relativo.

Tabela 2. Composição química do óleo de soja virgem e de fritura

\begin{tabular}{|c|c|c|c|c|c|c|c|c|c|}
\hline \multicolumn{10}{|c|}{ Teor de ésteres $(\%)$} \\
\hline \multirow{2}{*}{ Período (dias) } & \multicolumn{9}{|c|}{ Biodiesel de óleo virgem } \\
\hline & Plástico & D.P. ${ }^{a}$ & Rep. $^{\text {b }}$ & Vidro & D.P. ${ }^{\mathrm{a}}$ & Rep. $^{\text {b }}$ & Lata & D.P. ${ }^{a}$ & Rep. $^{\text {b }}$ \\
\hline 0 & $<96,5^{*}$ & & & & & & & & \\
\hline 30 & 93,50 & - & - & 92,75 & 0,35 & 0,18 & 81,95 & 0,21 & 0,17 \\
\hline 60 & 92,70 & 1,48 & 0,18 & 94,90 & 1,27 & 0,19 & 75,00 & 1,27 & 0,15 \\
\hline 120 & $<96,5$ & - & - & $<96,5$ & - & - & 58,50 & - & - \\
\hline 150 & 91,90 & - & - & $<96,5$ & 9,26 & 0,20 & 49,40 & 2,12 & 0,11 \\
\hline 180 & 96,50 & 1,09 & 0,19 & 96,05 & 0,49 & 0,19 & 48,80 & 1,13 & 0,11 \\
\hline \multirow{2}{*}{ Período (dias) } & \multicolumn{9}{|c|}{ Biodiesel de óleo de fritura } \\
\hline & Plástico & D.P. ${ }^{a}$ & Rep. $^{b}$ & Vidro & D.P. ${ }^{a}$ & Rep. $^{\text {b }}$ & Lata & D.P. ${ }^{a}$ & Rep. $^{b}$ \\
\hline 0 & $<96,5^{*}$ & & & & & & & & \\
\hline 30 & 91,80 & 0,85 & 0,18 & 92,60 & 6,08 & 0,18 & 86,50 & 3,04 & 0,17 \\
\hline 60 & 92,80 & 0,14 & 0,18 & 94,10 & 0,92 & 0,19 & 78,10 & 0,07 & 0,16 \\
\hline 120 & 96,00 & - & - & $<96,5$ & - & - & 66,00 & - & - \\
\hline 150 & 93,20 & - & - & 91,20 & 3,90 & 0,18 & 69,20 & 2,54 & 0,14 \\
\hline 180 & 95,20 & 1,13 & 0,19 & 96,50 & 2,12 & 0,19 & 61,50 & 6,57 & 0,13 \\
\hline
\end{tabular}

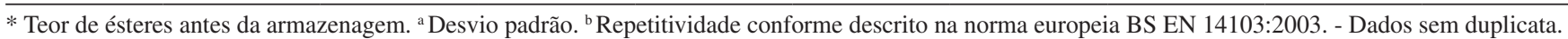


Tabela 3. Avaliação da atmosfera oxidativa no teor de ésteres do biodiesel de óleo de caixa de gordura

\begin{tabular}{|c|c|c|c|c|c|c|}
\hline \multicolumn{7}{|c|}{ Teor de ésteres $(\%)$} \\
\hline \multirow{2}{*}{ Período (dias) } & \multicolumn{6}{|c|}{ Recipiente de plástico } \\
\hline & Aberto & D.P. ${ }^{a}$ & Rep. $^{\text {b }}$ & Fechado & D.P. ${ }^{a}$ & Rep. $^{\mathrm{b}}$ \\
\hline 0 & $92,60 *$ & & & & & \\
\hline 60 & 80,00 & - & - & 82,00 & 2,54 & 0,17 \\
\hline 90 & 73,00 & - & - & 77,00 & - & - \\
\hline 120 & 75,40 & 3,11 & 0,15 & 78,15 & 5,73 & 0,16 \\
\hline 150 & 69,30 & - & - & 74,30 & 0,14 & 0,15 \\
\hline 210 & 66,50 & - & - & 71,10 & 0,14 & 0,15 \\
\hline 240 & 67,30 & 0,71 & 0,14 & 72,90 & 1,83 & 0,15 \\
\hline 270 & 62,97 & 0,19 & 0,13 & 76,73 & 2,26 & 0,16 \\
\hline \multirow[t]{3}{*}{360} & 62,30 & 0,42 & 0,13 & 72,70 & 3,96 & 0,15 \\
\hline & \multicolumn{6}{|c|}{ Recipiente de vidro } \\
\hline & Aberto & D.P. ${ }^{a}$ & Rep. $^{\text {b }}$ & Fechado & D.P. ${ }^{a}$ & Rep. $^{\text {b }}$ \\
\hline \multicolumn{7}{|l|}{0} \\
\hline 60 & 78,00 & 2,54 & 0,16 & 83,00 & 0,35 & 0,17 \\
\hline 90 & 73,70 & - & - & 77,60 & - & - \\
\hline 120 & 74,30 & 0,28 & 0,15 & 77,40 & 1,83 & 0,16 \\
\hline 150 & 75,00 & - & - & 71,35 & 1,48 & 0,15 \\
\hline 210 & 66,75 & 0,21 & 0,14 & 77,60 & 0,71 & 0,16 \\
\hline 240 & 68,45 & 1,20 & 0,14 & 78,15 & 0,07 & 0,16 \\
\hline 270 & 64,40 & 0,67 & 0,13 & 76,54 & 0,66 & 0,15 \\
\hline \multirow[t]{3}{*}{360} & 61,55 & 0,50 & 0,13 & 77,60 & 1,84 & 0,16 \\
\hline & \multicolumn{6}{|c|}{ Recipiente de lata } \\
\hline & Aberto & D.P. ${ }^{a}$ & Rep. $^{\text {b }}$ & Fechado & D.P. ${ }^{a}$ & Rep. $^{\text {b }}$ \\
\hline \multicolumn{7}{|l|}{0} \\
\hline 60 & 83,00 & 3,04 & 0,17 & 80,00 & 3,67 & 0,16 \\
\hline 90 & 74,50 & - & - & 75,95 & 0,35 & 0,15 \\
\hline 120 & 72,35 & 0,21 & 0,15 & 77,85 & 1,2 & 0,16 \\
\hline 150 & 68,15 & 0,07 & 0,14 & 78,05 & 0,49 & 0,16 \\
\hline 210 & 60,65 & 0,35 & 0,13 & 76,55 & 0,35 & 0,15 \\
\hline 240 & 58,65 & 0,63 & 0,13 & 75,60 & 0,56 & 0,15 \\
\hline 270 & 51,50 & 0,25 & 0,11 & 75,48 & 0,41 & 0,15 \\
\hline 360 & 44,90 & - & - & - & - & - \\
\hline
\end{tabular}

*Teor de ésteres antes da armazenagem. ${ }^{a}$ Desvio padrão. ${ }^{b}$ Repetitividade conforme descrito na norma BS EN 14103:2003. - Dados sem duplicata.

\section{Índice de acidez}

O índice de acidez e a estabilidade à oxidação foram os primeiros ensaios realizados, para evitar o contato das amostras com o oxigênio e com a umidade do ar. Leung e colaboradores (2006) avaliaram o índice de acidez de biodiesel de óleo de colza sob diferentes condições e comprovaram que a temperatura, a presença de água e o ar atmosférico, aumentam o índice de acidez, o que indica uma maior taxa de degradação da amostra. ${ }^{35}$

Para as amostras de biodiesel de óleo de soja virgem e de fritura, o índice de acidez inicial foi 0,14 e $0,13 \mathrm{mg} \mathrm{KOH} \mathrm{g}^{-1}$, respectivamente. Com o passar do tempo de estocagem, verificou-se que para ambos os biodieseis, armazenados em frascos de vidro ou plástico, as alterações no índice de acidez obtiveram pouca variação, em até $0,1 \mathrm{mg} \mathrm{KOH} \mathrm{g}^{-1}$. Já para os mesmos biodieseis estocados em lata, as mudanças ficaram acima de 4,0 $\mathrm{mg} \mathrm{KOH} \mathrm{g}^{-1}$ (Tabela 4).

$\mathrm{O}$ efeito catalisador dos metais da lata foi provavelmente um dos principais responsáveis pela degradação dos ésteres, que ao reagirem liberam ácidos graxos livres aumentando o teor de acidez. A partir de sessenta dias de estocagem, os biodieseis acondicionados em lata apresentaram índice de acidez fora da especificação, fazendo com que esta propriedade não fosse mais monitorada. De acordo com a legislação vigente, o índice de acidez do biodiesel deve ser de até 0,5 $\mathrm{mg} \mathrm{KOH} \mathrm{g}{ }^{-1}$. Altos valores de índice de acidez no biodiesel sinalizam o comprometimento em sua qualidade e são, portanto, indesejáveis, uma vez que podem acarretar uma ação corrosiva no motor. ${ }^{36,37}$

O índice de acidez do biodiesel de óleo de caixa de gordura se manteve dentro da especificação por noventa dias de estocagem. Após este tempo, todas as amostras aumentaram seu valor de acidez, independente da condição de armazenagem. É notável um crescimento maior na acidez dos biodieseis estocados nos frascos abertos, em contato com a atmosfera oxidativa, comparado com os que foram mantidos em recipientes fechados sob atmosfera inerte (Figura 1).

Após duzentos e quarenta dias de armazenamento, o biodiesel de óleo de caixa de gordura acondicionado em lata aberta com acidez inicial de 0,22 $\mathrm{mg} \mathrm{KOH} \mathrm{g}^{-1}$ passou a apresentar o índice de acidez igual a $17 \mathrm{mg} \mathrm{KOH} \mathrm{g}^{-1}$, com isso, o teor de ésteres também sofreu uma diminuição notável, de 92,6\% para 45,0\%, devido a degradação. Pressupõe-se, com base na literatura, que isso ocorreu porque estes ésteres foram mantidos em um ambiente favorável à hidrólise (lata, com presença de atmosfera oxidativa), o qual levou à formação de ácidos graxos livres. Por meio da derivatização e análise por CG$\mathrm{EM}$, o teor relativo de ácidos graxos livres foi revelado, totalizando $34,34 \%$, que corresponde a $15,50 \%$ de ácido palmítico, $12,38 \%$ de 
Tabela 4. Índice de acidez do biodiesel de óleo de soja virgem e de fritura

\begin{tabular}{|c|c|c|c|c|c|c|c|c|c|}
\hline \multicolumn{10}{|c|}{ Índice de acidez (mg KOH g $\left.{ }^{-1}\right)$} \\
\hline \multirow{2}{*}{ Período (dias) } & \multicolumn{9}{|c|}{ Biodiesel de óleo de soja } \\
\hline & Plástico & D.P. ${ }^{\mathrm{a}}$ & Rep. $^{\mathrm{b}}$ & Vidro & D.P. ${ }^{a}$ & Rep. $^{\text {b }}$ & Lata & D.P. ${ }^{a}$ & Rep. $^{\text {b }}$ \\
\hline 0 & 0,14 & - & - & 0,14 & & & 0,14 & - & \\
\hline 30 & 0,12 & 0,02 & 0,049 & 0,13 & 0 & 0,05 & 0,72 & $7,1 \times 10^{-5}$ & 0,075 \\
\hline 60 & 0,15 & - & - & 0,15 & 0,003 & 0,05 & 2,75 & - & - \\
\hline 120 & 0,13 & - & - & 0,08 & - & - & 5,62 & - & - \\
\hline 150 & 0,10 & $7,1 \times 10^{-5}$ & 0,048 & 0,23 & - & - & - & - & - \\
\hline 180 & 0,15 & - & - & 0,08 & - & - & - & - & - \\
\hline \multirow{2}{*}{ Período (dias) } & \multicolumn{9}{|c|}{ Biodiesel de óleo de fritura } \\
\hline & Plástico & D.P. ${ }^{a}$ & Rep. $^{b}$ & Vidro & D.P. ${ }^{a}$ & Rep. $^{\text {b }}$ & Lata & D.P. ${ }^{a}$ & Rep. $^{\text {b }}$ \\
\hline 0 & 0,13 & & & 0,13 & & & 0,13 & & \\
\hline 30 & 0,18 & - & - & 0,15 & 0 & 0,05 & 0,38 & 0,03 & 0,06 \\
\hline 60 & 0,08 & - & - & 0,05 & 0 & 0,046 & 2,27 & - & - \\
\hline 120 & 0,23 & - & - & 0,05 & - & - & 2,80 & - & - \\
\hline 150 & 0,20 & - & - & 0,08 & - & - & 4,89 & - & - \\
\hline 180 & 0,13 & - & - & 0,05 & 0 & 0,046 & - & - & - \\
\hline
\end{tabular}

${ }^{\mathrm{a}}$ Desvio Padrão. ${ }^{\mathrm{b}}$ Repetitividade conforme descrito na norma ABNT NBR 14448:2009. - Representa os resultados sem duplicata ou não realizados nas amostras já degradadas.

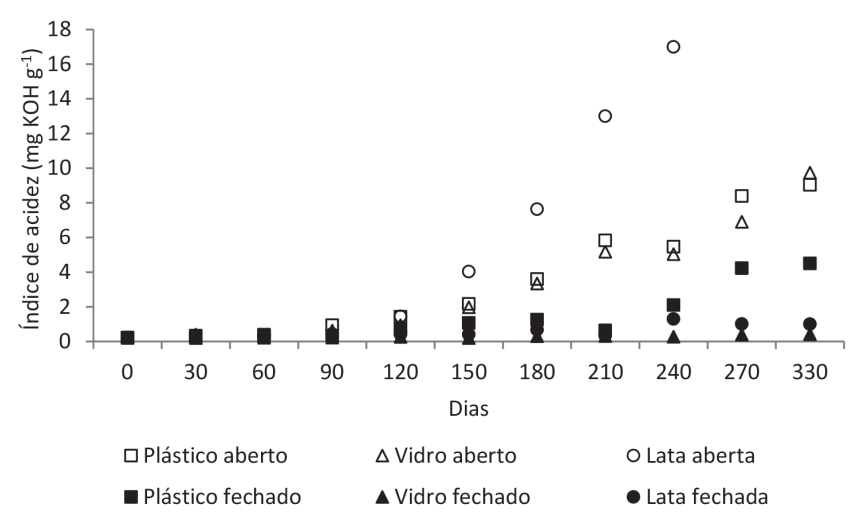

Figura 1. Variação da acidez de biodiesel de óleo de caixa de gordura armazenado em condições diferenciadas

ácido linoleico e 6,46\% de ácido esteárico.

\section{Índice de iodo}

O índice de iodo está diretamente relacionado com a estabilidade à oxidação e fornece o teor de insaturação de uma amostra. Todavia, este ensaio por si só não pode predizer a estabilidade do biodiesel, uma vez que quantifica as ligações duplas sem distinção da sua posição na molécula, o que é substancial em termos de reatividade. Assim, o índice de iodo é importante para confirmar, juntamente com os outros testes, se as amostras de biodiesel têm a possibilidade de formar subprodutos lipídicos, os quais poderão causar entupimento em um motor. ${ }^{15}$

No Brasil, não há exigência quanto aos limites de índice de iodo para o biodiesel, de modo que a legislação vigente solicita apenas a averiguação deste parâmetro (Resolução ANP Nº 14 de 11.05.2012). Pela Norma Europeia, o índice de iodo deve estar próximo de $120 \mathrm{~g} \mathrm{I}_{2} 100 \mathrm{~g}^{-1}$.

As amostras de biodiesel de óleo de soja virgem e de fritura apresentaram valores de iodo iniciais muito similares, sendo $123,70 \mathrm{~g} \mathrm{I}_{2}$ $100 \mathrm{~g}^{-1}$ e 123,00 $\mathrm{g} \mathrm{I}_{2} 100 \mathrm{~g}^{-1}$, respectivamente. Com o passar do tempo, ambas sofreram diminuição do índice de iodo (Tabela 5), sendo que as amostras armazenadas em latas tiveram reduções mais significativas,
Tabela 5. Índice de iodo de biodiesel de óleo virgem e de fritura

\begin{tabular}{lcccccc}
\hline \multicolumn{7}{c}{ Índice de iodo $\left(\mathrm{g} \mathrm{I}_{2} 100 \mathrm{~g}^{-1}\right)$} \\
\hline $\begin{array}{l}\text { Período } \\
\text { dias })\end{array}$ & \multicolumn{3}{c}{ Biodiesel de soja virgem } & \multicolumn{3}{c}{ Biodiesel de fritura } \\
\cline { 2 - 7 } & Plástico & Vidro & Lata & Plástico & Vidro & Lata \\
\hline 0 & 123,70 & 123,70 & 123,70 & 123,00 & 123,00 & 123,00 \\
30 & 131,50 & 107,00 & 101,05 & 128,44 & 129,35 & 127,58 \\
60 & 108,00 & 110,40 & 97,40 & 106,00 & 105,00 & 100,00 \\
120 & 92,12 & 109,03 & 75,85 & 112,11 & 107,71 & 94,55 \\
150 & 91,73 & 114,14 & 36,44 & 103,41 & 113,51 & 77,10 \\
180 & 114,00 & 115,41 & 73,70 & 115,67 & 119,00 & 81,96 \\
\hline
\end{tabular}

causadas pela perda das duplas ligações. Esse efeito pode ser mais uma vez atribuído ao efeito catalítico dos metais constituintes do recipiente, que favorecem a ocorrência de reações químicas.

O índice de iodo também varia com o tipo de matéria-prima empregada na produção de biodiesel, devido à composição dos triacilgliceróis em cada matriz. ${ }^{38,39}$ Por este motivo, é possível notar que os índices de iodo dos biodieseis de óleo de soja virgem e de fritura diferem do biodiesel oriundo do óleo de caixa de gordura. Observando a variação do índice de iodo deste biodiesel sob condições de armazenamento distintas, foi notada uma maior preservação das insaturações para a amostra armazenada em vidro fechado. Seu comportamento está mostrado na Figura 2.

O biodiesel de óleo de caixa de gordura mostrou um comportamento similar aos biodieseis produzidos neste trabalho. Verificou-se que sob todas as condições de armazenamento, houve um aumento do índice de iodo seguido de leve queda. Isso evidencia que a composição química desse material está em constante transformação.

\section{Viscosidade cinemática}

A viscosidade cinemática é a medida da resistência ao escoamento e é considerada uma importante propriedade dos combustíveis, pois pode comprometer o desempenho do motor. ${ }^{40}$ Os óleos vegetais são transformados em biodiesel para que possuam uma menor viscosidade e melhorem a atomização, o que evita depósitos, desgaste do motor e elevado consumo de energia. ${ }^{41,42}$ 


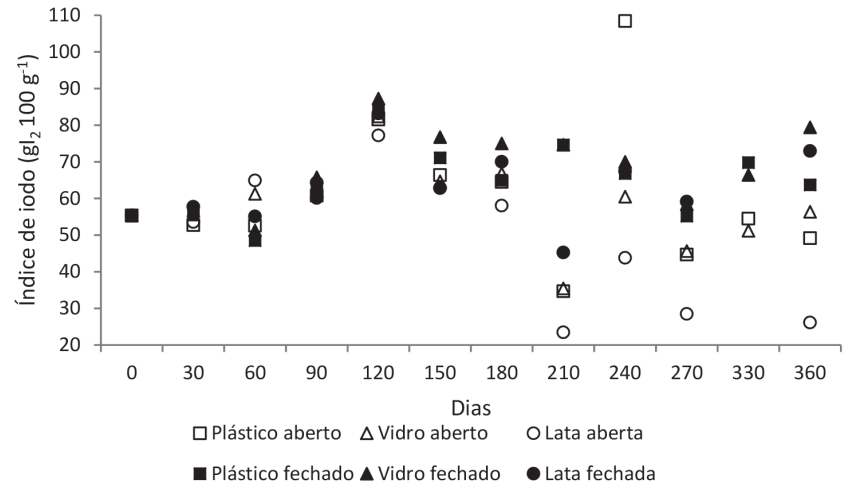

Figura 2. Índice de iodo do biodiesel de óleo de caixa de gordura

A viscosidade do biodiesel é maior com o aumento da cadeia de carbonos, grau de insaturação do triacilglicerol e presença de ácidos graxos livres. Amostras insaturadas com configuração cis são mais viscosas que aquelas com configuração trans. Já a posição da dupla ligação não interfere tão significativamente na viscosidade. ${ }^{43}$

Os resultados dessa pesquisa mostraram que a viscosidade se manteve com pouca variação, tanto para o biodiesel de óleo de soja virgem, quanto para o biodiesel de óleo de fritura armazenado em recipiente de plástico e vidro. Ao longo de duzentos e dez dias de armazenamento, a média da viscosidade cinemática medida a 40 ${ }^{\circ} \mathrm{C}$ para o biodiesel de óleo de soja virgem armazenado em plástico e em vidro ficou $3,784 \mathrm{~mm}^{2} \mathrm{~s}^{-1}$ e $3,674 \mathrm{~mm}^{2} \mathrm{~s}^{-1}$, respectivamente. Para o biodiesel de óleo de fritura, a média para este mesmo período e condição de armazenamento foi similar, sendo $3,993 \mathrm{~mm}^{2}$ $\mathrm{s}^{-1}$ para o recipiente de plástico e $3,873 \mathrm{~mm}^{2} \mathrm{~s}^{-1}$ para o estocado em vidro. Estes valores estão em conformidade com a legislação brasileira, que estipula a faixa de viscosidade para o biodiesel entre 3,0 e $6,0 \mathrm{~mm}^{2} \mathrm{~s}^{-1}$ a $40{ }^{\circ} \mathrm{C}$ (Resolução ANP N ${ }^{\circ} 14$ de 11.05.2012). $\mathrm{O}$ biodiesel de óleo de caixa de gordura também apresentou uma viscosidade estável durante o período de armazenamento em plástico e vidro. Embora a viscosidade deste biocombustível não tenha sido significativamente afetada ao ser submetido às condições de armazenagem descritas, não é possível afirmar que o vidro e o plástico são materiais adequados para a estocagem de biodiesel, uma vez que outras características devem ser avaliadas tornando o estudo mais conclusivo.

Os biodieseis armazenados em lata tiveram aumento considerável nas suas viscosidades. $\mathrm{O}$ efeito catalisador dos metais é o principal responsável por esse resultado, uma vez que os metais presentes na lata desencadeiam reações químicas que levam à formação de produtos de polimerização, os quais aumentam a viscosidade do biocombustível e comprometem sua estabilidade à oxidação. ${ }^{39,43}$ Até mesmo em baixas concentrações a presença dos metais é indesejável no biodiesel. ${ }^{20,44}$ Como pode ser visualizado na Figura 3, os três tipos de biodiesel estudados apresentaram alterações nas suas viscosidades, devido ao acondicionamento em embalagem metálica. Ainda deve ser considerada a condição diferenciada da capacidade da lata metálica, com um "headspace" de $400 \mathrm{~mL}$. A Figura 3 também mostra que, nos primeiros noventa dias, as amostras de biodiesel armazenadas em lata se mantiveram mais preservadas, no entanto, após este período, as alterações na viscosidade se intensificaram e ultrapassaram o limite máximo estipulado pela legislação brasileira, que é de $6 \mathrm{~mm}^{2} \mathrm{~s}^{-1}$.

\section{Estabilidade à oxidação}

A oxidação pode ser promovida por temperatura elevada, presença de materiais estranhos, tais como metais, condições de estocagem, absorção de água, presença de microrganismos, por auto oxidação

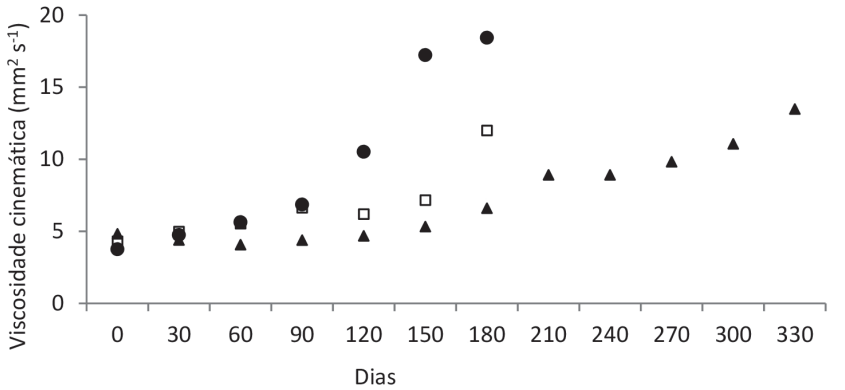

\section{- Biodiesel de óleo virgem \\ $\Delta$ Biodiesel de caixa de gordura}

$\square$ Biodiesel de óleo de fritura

Figura 3. Viscosidade de biodiesel armazenado em lata

natural e pelo contato com ar. ${ }^{20,45}$ Analisando as condições de estocagem do biodiesel de óleo de caixa de gordura (em plástico, lata, vidro, na presença e ausência de nitrogênio), verificou-se que somente a amostra armazenada em frasco de vidro em presença de nitrogênio se manteve preservada por mais tempo (Figura 4, Tabela 6), sendo, $7,420 \mathrm{~h}$ em seu tempo inicial e 2,890 h após trezentos e sessenta dias. As demais amostras apresentaram poucos minutos de estabilidade.

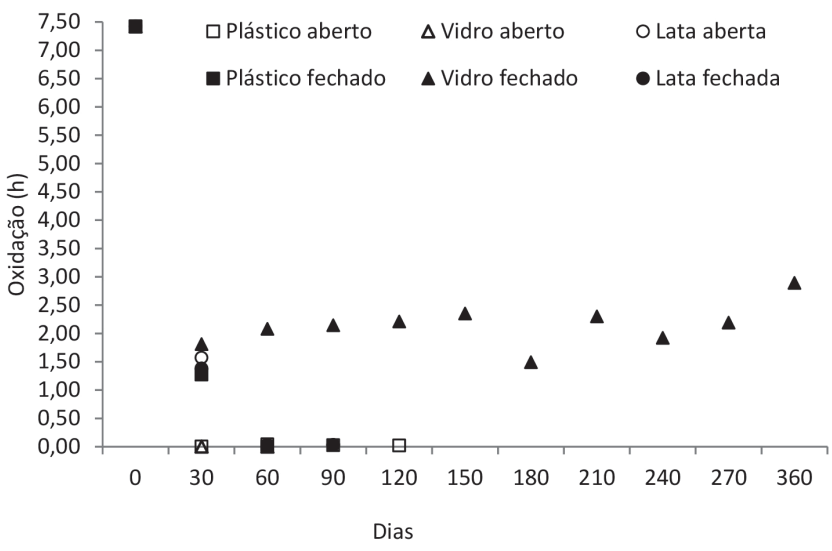

Figura 4. Estabilidade à oxidação em condições de estocagem diferenciadas

A oxidação dos biodieseis analisados pode ser atribuída a fatores conhecidos teoricamente, contudo, alguns ensaios realizados permitiram inferir que as causas da oxidação foram a natureza insaturada do triacilglicerol, o contato com atmosfera oxidativa e a estocagem em reservatório metálico com ação catalítica. Outros testes como a determinação do teor de água e os ensaios microbiológicos ainda podem ser realizados para melhorar a compreensão sobre os motivos da oxidação. Pela legislação brasileira, a estabilidade à oxidação mínima deve ser de 6 horas a $110^{\circ} \mathrm{C}$, o que leva a aconselhar que a utilização deste biodiesel deve ser imediata, caso não sejam adicionados antioxidantes, dado que longos períodos de estocagem contribuem para a sua degradação. ${ }^{46}$

\section{Avaliação da estabilidade das misturas}

As misturas foram obtidas por meio da adição de biodiesel de óleo de fritura com biodiesel de óleo virgem, nas proporções de $1 \%$, $5 \%$ e $10 \%(\mathrm{~m} / \mathrm{m})$. Os resultados da avaliação das propriedades das misturas no início e após sessenta dias de estocagem estão apresentados na Tabela 7.

Ao longo do período de estocagem notou-se variação nas três propriedades das misturas, no entanto, o índice de acidez se manteve 
Tabela 6. Estabilidade à oxidação de biodiesel de caixa de gordura

\begin{tabular}{|c|c|c|c|c|c|c|}
\hline \multicolumn{7}{|c|}{ Estabilidade à oxidação (h) } \\
\hline \multirow{2}{*}{$\begin{array}{l}\text { Período } \\
\text { (dias) }\end{array}$} & \multicolumn{6}{|c|}{ Recipiente de plástico } \\
\hline & Aberto & D.P. ${ }^{a}$ & Rep. $^{b}$ & Fechado & D.P. ${ }^{a}$ & Rep. $^{\text {b }}$ \\
\hline 0 & $7,420^{*}$ & & & & & \\
\hline 30 & 0 & - & - & 1,270 & - & - \\
\hline 60 & 0,040 & 0 & 0,164 & 0 & - & - \\
\hline 90 & 0,025 & 0,007 & 0,162 & - & - & - \\
\hline \multirow[t]{3}{*}{120} & 0,020 & 0 & 0,162 & - & - & - \\
\hline & \multicolumn{6}{|c|}{ Recipiente de vidro } \\
\hline & Aberto & D.P. ${ }^{a}$ & Rep. $^{\text {b }}$ & Fechado & D.P. ${ }^{a}$ & Rep. $^{\text {b }}$ \\
\hline 0 & $7,420^{*}$ & & & & & \\
\hline 30 & 0 & - & - & 1,810 & - & - \\
\hline 60 & 0 & 0 & 0,160 & 2,080 & 0 & 0,347 \\
\hline 90 & - & - & - & 2,145 & 0,035 & 0,353 \\
\hline 120 & - & - & - & 2,210 & 0 & 0,359 \\
\hline 150 & - & - & - & 2,350 & 0,290 & 0,351 \\
\hline 180 & - & - & - & 1,490 & - & - \\
\hline 210 & - & - & - & 2,300 & 0,110 & 0,367 \\
\hline 240 & - & - & - & 1,920 & 0,042 & 0,333 \\
\hline 270 & & - & - & 2,190 & - & - \\
\hline 360 & - & & & 2,890 & - & - \\
\hline
\end{tabular}

\begin{tabular}{lcccccc}
\hline & \multicolumn{6}{c}{ Recipiente de lata } \\
\cline { 2 - 7 } & Aberto & D.P. $^{\text {a }}$ & Rep. $^{\text {b }}$ & Fechado & D.P. $^{\text {a }}$ & Rep. $^{\text {b }}$ \\
\hline 0 & $7,420^{*}$ & & & & & \\
30 & 1,570 & - & - & 1,380 & - & - \\
60 & 0,035 & 0 & 1,63 & 0 & 0 & 0,160 \\
90 & 0,030 & 0 & 1,63 & 0,030 & 0 & 0,163 \\
\hline
\end{tabular}

${ }^{a}$ Desvio Padrão. ${ }^{b}$ Repetitividade conforme descrito na norma europeia BS EN 14112:2003. - Representa os resultados sem duplicata ou não realizados nas amostras já degradadas. *Resultado antes da armazenagem.

Tabela 7. Avaliação das propriedades das misturas

\begin{tabular}{|c|c|c|c|c|c|c|}
\hline \multirow[t]{2}{*}{ Amostras } & \multicolumn{2}{|c|}{$\begin{array}{l}\text { Índice de acidez } \\
\left(\mathrm{mg} \mathrm{KOH} \mathrm{g}^{-1}\right)\end{array}$} & \multicolumn{2}{|c|}{$\begin{array}{l}\text { Estabilidade a } \\
\text { oxidação (h) }\end{array}$} & \multicolumn{2}{|c|}{$\begin{array}{c}\text { Teor de ésteres } \\
(\%)\end{array}$} \\
\hline & Início & Fim & Início & Fim & Início & Fim \\
\hline B.O.V. ${ }^{a}$ & 0,60 & 0,78 & 3,68 & 3,47 & 99,6 & 95,00 \\
\hline B.O.F. ${ }^{b}$ & 1,50 & 1,60 & 3,28 & 2,90 & 96,4 & 88,40 \\
\hline Mistura $1 \%$ & 0,78 & 0,78 & 3,66 & 3,35 & 98,00 & 91,30 \\
\hline Mistura 5\% & 0,78 & 0,78 & 3,76 & 2,96 & 97,00 & 92,10 \\
\hline Mistura $10 \%$ & 0,78 & 0,78 & 4,25 & 3,58 & 96,00 & 94,50 \\
\hline
\end{tabular}

${ }^{\mathrm{a}}$ biodiesel de óleo virgem. ${ }^{\mathrm{b}}$ biodiesel de óleo de fritura.

praticamente constante. Houve diminuição significativa na estabilidade à oxidação e no teor de ésteres, porém, deve-se considerar que estas misturas não possuem nenhum tipo de conservante, que se empregado, poderia diminuir a degradação deste biocombustível. A utilização de biodiesel de óleo de fritura em misturas pode ser uma alternativa interessante do ponto de vista econômico e ecológico.

\section{CONCLUSÃO}

Biodieseis oriundos de óleo de fritura e óleo de soja virgem foram produzidos neste trabalho, empregando transesterificação básica com metanol, que promoveu a conversão de ésteres superior ao mínimo exigido pela especificação. A partir desses dois biodieseis, foram preparadas misturas com proporções mássicas de $1 \%, 5 \%$ e $10 \%$ de biodiesel de óleo de fritura diluído no biodiesel de óleo de soja virgem. Um biodiesel de óleo de caixa de gordura também foi avaliado neste estudo. Tanto os biodieseis na forma pura (provenientes de óleo virgem, oléo de fritura e caixa de gordura) como as misturas preparadas foram armazenados em condições diferenciadas e tiveram seus parâmetros físico-químicos indicativos de estabilidade monitorados ao longo do tempo. Entre estes parâmetros estão o índice de iodo, a viscosidade, o índice de acidez e o teor de ésteres que, ao serem averiguados, evidenciaram que as condições de armazenamento são cruciais para a manutenção das propriedades do biodiesel. A estocagem em lata em contato com o ar atmosférico não é aconselhável, devido ao efeito catalítico dos metais na degradação do biocombustível, fato que levou a um reduzido teor de ésteres (48,8\% para o biodiesel de óleo de soja virgem e $61,5 \%$ para o óleo de fritura). Em consequência da degradação dos ésteres, houve um aumento no índice de acidez, que, após noventa dias de estocagem em lata, sobressaiu os limites aceitáveis pela legislação vigente. A viscosidade do biodiesel armazenado em lata também foi afetada de maneira negativa, indicando a baixa qualidade do combustível ao final do período avaliado. Este estudo também revelou que o armazenamento em plástico e em vidro é mais adequado e pode ser melhorado quando submetido à atmosfera de nitrogênio.

O monitoramento do índice de acidez, da estabilidade à oxidação e do teor de ésteres das misturas apontou a possibilidade de utilização imediata de biodiesel de óleo de fritura misturado ao biodiesel de óleo virgem em proporções de até $5 \%(\mathrm{~m} / \mathrm{m})$. Os resultados sugerem que deve ser feita a adição de antioxidantes para evitar a deterioração dos ésteres.

\section{AGRADECIMENTOS}

Os autores agradecem à CAPES pela bolsa de pesquisa e ao CNPq (Processo 558724/2010-8) pelo apoio financeiro. Os autores também agradecem a ECO 100 Desenvolvimento Sustentado Ltda e a Companhia Estadual de Águas e Esgotos-CEDAE da cidade do Rio de Janeiro pelo fornecimento do produto testado neste trabalho.

\section{REFERÊNCIAS}

1. Santori, G.; Di Nicola, G.; Moglie, M.; Polonara, F.; Appl. Energy 2012, 92, 109.

2. Ramos, L. P.; Kothe, V.; César-Oliveira, M. A. F.; Muniz-Wypych, A. S.; Nakagaki, S.; Krieger, N.; Wypych, F.; Cordeiro, C. S.; Rev. Virtual Quim. 2017, 9, 317.

3. Ramos, L. P.; Silva, F. R.; Mangrich, A. S.; Cordeiro, S.; Kothe, V.; César-Oliveira, M. A. F.; Muniz-Wypych, A. S.; Nakagaki, S.; Krieger, N.; Wypych, F.; Cordeiro, C. S.; Rev. Virtual Quim. 2011, 3, 385.

4. Ramachandran, K.; Sivakumar, P.; Suganya, T.; Renganathan, S.; Bioresour. Technol. 2011, 102, 7289.

5. Cunha, A.; Feddern, V.; De Prá, M. C.; Higarashi, M. M.; de Abreu, P. G.; Coldebella, A.; Fuel 2013, 105, 228.

6. Choi, O. K.; Song, J. S.; Cha, D. K.; Lee, J. W.; Bioresour. Technol. 2014, 166, 51.

7. Silva, T. A.; de Assunção, R. M. N.; Vieira, A. T.; de Oliveira, M. F.; Batista, A. C. F.; Fuel 2014, 136, 10. 
8. Silva, G. S.; Marques, E. L. S.; Dias, J. C. T.; Lobo, I. P.; Gross, E.; Brendel, M.; Da Cruz, R. S.; Rezende, R. P.; Appl. Soil Ecol. 2012, 55, 27.

9. Kleinová, A.; Cvengrošová, Z.; Cvengroš, J.; Fuel 2013, 109, 588.

10. Jain, S.; Sharma, M. P.; Renewable Sustainable Energy Rev. 2011, 15, 438.

11. Freire, L. M. D. S.; Santos, I. M. G. Dos; De Carvalho Filho, J. R.; Cordeiro, A. M. T. D. M.; Soledade, L. E. B.; Fernandes, V. J.; De Araujo, A. S.; De Souza, A. G.; Fuel 2012, 94, 313.

12. Demirbas, A.; Energy Convers. Manage. 2009, 50, 923.

13. Bankovi -Ili, I. B.; Stamenkovi, O. S.; Veljkovi, V. B.; Renewable Sustainable Energy Rev. 2012, 16, 3621.

14. Ashraful, A. M.; Masjuki, H. H.; Kalam, M. A.; Rizwanul Fattah, I. M.; Imtenan, S.; Shahir, S. A.; Mobarak, H. M.; Energy Convers. Manage. 2014, 80, 202.

15. Pullen, J.; Saeed, K.; Renewable Sustainable Energy Rev. 2012, 16 , 5924.

16. Berrios, M.; Martín, M. A.; Chica, A. F.; Martín, A.; Fuel 2012, 91, 119.

17. Dantas, M. B.; Albuquerque, A. R.; Barros, A. K.; Rodrigues Filho, M. G.; Antoniosi Filho, N. R.; Sinfrônio, F. S. M.; Rosenhaim, R.; Soledade, L. E. B.; Santos, I. M. G.; Souza, A. G.; Fuel 2011, 90, 773.

18. Thompson, M. R.; Mu, B.; Ewaschuk, C. M.; Cai, Y.; Oxby, K. J.; Vlachopoulos, J.; Fuel 2013, 108, 771.

19. Na-Ranong, D.; Kitchaiya, P.; Fuel 2014, 122, 287.

20. Jakeria, M. R.; Fazal, M. A.; Haseeb, A. S. M. A.; Renewable Sustainable Energy Rev. 2014, 30, 154.

21. Fazal, M. A.; Jakeria, M. R.; Haseeb, A. S. M. A.; Rubaiee, S.; Energy 2017, 135, 220.

22. Karavalakis, G.; Hilari, D.; Givalou, L.; Karonis, D.; Stournas, S.; Energy 2011, 36, 369.

23. García, M.; Botella, L.; Gil-Lalaguna, N.; Arauzo, J.; Gonzalo, A.; Sánchez, J. L.; Fuel Process. Technol. 2017, 156, 407.

24. Varatharajan, K.; Pushparani, D. S.; Renewable Sustainable Energy Rev. 2018, 82, 2017.

25. Agarwal, A. K.; Khurana, D.; Fuel Process. Technol. 2013, 106, 447.
26. Pinho, D. M. M.; Santos, V. O.; dos Santos, V. M. L.; Oliveira, M. C. S.; da Silva, M. T.; Piza, P. G. T.; Pinto, A. C.; Rezende, M. J. C.; Suarez, P. A. Z.; Fuel 2014, 136, 136.

27. Botella, L.; Bimbela, F.; Martín, L.; Arauzo, J.; Sánchez, J. L.; Front. Chem. 2014, 2, 1.

28. Christensen, E.; McCormick, R. L. Fuel Process. Technol.; 2014, 128, 339.

29. Pullen, J.; Saeed, K.; Fuel Process. Technol. 2014, 125, 223.

30. Jain, S.; Sharma, M. P.; Renewable Sustainable Energy Rev. 2011, 15, 438.

31. Issariyakul, T.; Kulkarni, M. G.; Dalai, A. K.; Bakhshi, N. N.; Fuel Process. Technol. 2007, 88, 429.

32. Beheshti, S.; Turkish J. Chem. 2003, 27, 251.

33. Wisniewski, A.; Wiggers, V. R.; Simionatto, E. L.; Meier, H. F.; Barros, A. A. C.; Madureira, L. A. S.; Fuel 2010, 89, 563.

34. The Biodiesel Handbook; Knothe, G., Krahl, J., Van Gerpen, J., eds.; AOCS Press: Champaign, 2005; Vol. 1.

35. Leung, D. Y. C.; Koo, B. C. P.; Guo, Y.; Bioresour. Technol. 2006, 97, 250.

36. Demirbas, A. Energy Convers. Manage. 2008, 49, 125.

37. Albuquerque, G.; Dissertação de Mestrado, Universidade Federal de Pernambuco, Brasil, 2006, p. 100.

38. Hoekman, S. K.; Broch, A.; Robbins, C.; Ceniceros, E.; Natarajan, M.; Renewable Sustainable Energy Rev. 2012, 16, 143.

39. Yaakob, Z.; Narayanan, B. N.; Padikkaparambil, S.; Unni K. S.; Akbar P. M.; Renewable Sustainable Energy Rev. 2014, 35, 136.

40. Martinez, E. M.; Tormos, B.; Bermúdez, V.; Andrés, L.; Macián, V.; Tormos, B.; Bermúdez, V.; Ramírez, L.; Tribol. Int. 2014, 79, 132.

41. Maher, K. D.; Bressler, D. C.; Bioresour. Technol. 2007, 98, 2351.

42. Knothe, G.; Fuel Process. Technol. 2005, 86, 1059.

43. Knothe, G.; Steidley, K. R.; Fuel 2005, 84, 1059

44. Jain, S.; Sharma, M. P.; Fuel 2014, 116, 14.

45. Knothe, G.; Fuel Process. Technol. 2007, 88, 669.

46. Chen, Y.-H.; Luo, Y.-M.; Fuel Process. Technol. 2011, 92, 1387. 